Wright State University

CORE Scholar

$11-1-1994$

\title{
Interaction of a Group of Dislocations within the Framework of the Continuum Frenkel-Kontorova Model
}

Naum I. Gershenzon

Wright State University - Main Campus, naum.gershenzon@wright.edu

Follow this and additional works at: https://corescholar.libraries.wright.edu/physics

Part of the Earth Sciences Commons, Environmental Sciences Commons, and the Physics Commons

\section{Repository Citation}

Gershenzon, N. I. (1994). Interaction of a Group of Dislocations within the Framework of the Continuum Frenkel-Kontorova Model. Physical Review B, 50 (18), 13308-13314.

https://corescholar.libraries.wright.edu/physics/196

This Article is brought to you for free and open access by the Physics at CORE Scholar. It has been accepted for inclusion in Physics Faculty Publications by an authorized administrator of CORE Scholar. For more information, please contact library-corescholar@wright.edu. 


\title{
Interaction of a group of dislocations within the framework of the continuum Frenkel-Kontorova model
}

\author{
Naum I. Gershenzon \\ Wright State University, Dayton, Ohio 45435
}

(Received 15 June 1994)

\begin{abstract}
The Frenkel-Kontorova (FK) model of edge dislocation is analyzed. Solutions of the continuum limit of the FK model [the sine-Gordon (SG) equation] are obtained in a form convenient for investigation of dynamics of a large number of interacting dislocations. We consider, based on these solutions, some nonstationary processes: dislocation generation, diffusion of dislocations, and crack-dislocation interaction. Simple relations connecting the velocity of plastic deformation, density and velocity of dislocations, and the force of interaction between dislocations are obtained. The nucleation of dislocations at a moving crack tip is described.
\end{abstract}

\section{INTRODUCTION}

Processes of nucleation, movement, and interaction of dislocations play an important role in plasticity and destruction of crystal materials. ${ }^{1}$ The well-known model of edge dislocation introduced by Frenkel and Kontorova ${ }^{2}$ (FK) more then 50 years ago has been still widely investigated and applied for the crystal lattice ${ }^{3-10}$ and as universal theoretical model (see Refs. 11-13).

The FK model is a chain of atoms interacting via nearest-neighbor harmonic forces and placed in a periodic external potential. The simplest stable position of the chain corresponds to the perfect crystal. The configuration of $\mathbf{n}$ atoms, placed in $\mathbf{n}+\mathbf{1}$ (or $\mathbf{n}-\mathbf{1}$ ) periods of lattice, models positive (or negative) dislocation.

In the continuum limit the FK model is described by the one-dimensional Sine-Gordon (SG) equation. This equation has been intensely investigated with regards to a wide spectrum of applications. ${ }^{14}$ In application to the crystal lattice the basic solutions of the SG equation, kinks, breathers, and "plasma waves," are dislocations (or kinks on dislocation line), nonlinear oscillating modes, and phonons, respectively. Some discussion should be made of the use of the term "kink," since in the context of this paper this word is used to describe two different phenomena, one of which arises in the theory of the SG equation. The term "kink" was introduced as a special solution of SG equation. On the other hand, in the theory of dislocations, this term designates a kink of the dislocation line that is, where the dislocation is nonlinear, but is a curve with different lengths placed in the different Peierls value. ${ }^{1}$ Now, the SG equation can model either the straight-line dislocation propagating perpendicular to its axis, or, on the other hand, can model the propagation, in the direction along the dislocation, of a kink in the dislocation. Here we will only consider some examples with edge rectilinear dislocations moving in one slide plane perpendicular to the dislocation lines, although the developed theory could be also used for kinks.

The framework of the FK model and the SG equation has been used to model Peierls stress and energy, ${ }^{1,4-6}$ interaction of kinks and impurities, ${ }^{12}$ radiation of moved kinks, nucleation of kinks, influence of external stress, friction, and random force on the moving kinks, ${ }^{7,8,10,13}$ as well as formation of cracks. ${ }^{9}$

In the present paper we will consider some specific solutions of the SG equation by which will be obtained relations between macroscopic and microscopic parameters of plasticity. These solutions could be used for the description of nonstationary dynamic processes of group of interaction dislocations, in particular for a model of the generation of dislocations by source, diffusion of dislocations, and interaction between crack and dislocations.

We will limit our consideration of the SG equation to processes with slow changes of parameters in space and time. This restriction allows us to apply Whitham's variational method. ${ }^{15}$ This method is based on a definition of a system of modulation equations describing slow variations of the parameters of a wave train.

The paper is organized as follows. Section II contains the main proposition of the model. Using stationary solutions of the SG equation we determine a correspondence between parameters of the model and parameters describing dislocations and plasticity. The solutions of Whitham's equations based on the SG equation are obtained in Sec. III. Some examples of application of these solutions are considered in Sec. IV. Section V contains some concluding remarks.

\section{MODEL DESCRIPTION}

Attempts have been made to match the parameters of the FK model with the parameters of realistic crystal lattices. $^{2-6}$ Using these ideas, and restricting ourselves to the continuum limit, we can describe the movement of an atom chain in a periodic potential by the SG equation (here and below, the low indexes denote differentiation by the indicated variable):

$$
\varphi_{t t}-\varphi_{x x}+\sin \varphi=0
$$


where $\varphi(x, t)$ is the displacement of atoms relative to the "dislocationless" position measured in units of $b / 2 \pi ; x$ is a spatial coordinate in units of $b a_{x}$, where $a_{x}=2^{1 / 2} d / b(1-v)^{1 / 2} ; t$ is time in units of $b a_{t} / c_{l}$, where

$$
\begin{aligned}
& a_{t}=\frac{2^{1 / 2} d(1-v)^{1 / 2}}{b(1-2 v)^{1 / 2}}, \\
& c_{l}^{2}=\frac{2 \mu_{0} d c b(1-v)}{M(1-2 v)},
\end{aligned}
$$

$c_{l}$ is the velocity of sound in the crystal. Here, $b, c$, and $d$ are the lattice translations in $x, y$ (along the axis of dislocation) and $z$ (perpendicular to the glide-plane) directions, respectively; $M$ is the mass of an atom, $v$ is the Poisson ratio, $\mu_{0}$ is the shear modulus.

The variables $F=\varphi_{x}$, and $W=\varphi_{t}$ are the force of interaction between the nearest atoms and the velocity of the atoms, respectively: $F$ is measured in units of $b c a_{x} \sigma_{\mathrm{cr}}$, where $\sigma_{\mathrm{cr}}=\mu_{0} b / 2 \pi d$ is the amplitude of the Frenkel sinusoidal function, or the theoretical shear stress for inception of plastic deformation in the perfect crystal, $W$ is measured in units of $c_{l} / 2^{3 / 2} \pi a_{t}$.

The trivial solution $\varphi=0, F=0, W=0$ corresponds to a perfect crystal without dislocations. We will consider solutions of the SG equation of the form $\varphi=\varphi(\theta)$, that is, that depend on $x, t$ on through the variable $\theta=k \xi$, $\xi=x-U t$, where $k$ is the wave number, $\omega=k U$ is the frequency, and $U$ is the velocity of wave in units $c_{l} a_{x} / a_{t}$.

After the integration of Eq. (1) we obtain

$$
\theta=2^{-1 / 2} k\left(U^{2}-1\right)^{1 / 2} \int\left(A-V_{0}\right)^{-1 / 2} d \varphi,
$$

where $V_{0}=1-\cos \varphi$ and $A$ is the constant of integration. We will be interested only in periodic solutions, and so only consider the value of $\varphi$ between two zeros of the function $\left(A-V_{0}\right)$. Supposing in (2) $A=2 / m$ and $|U|<1$, we find the spiral waves solutions:

$$
\begin{aligned}
& \varphi=\arcsin [ \pm \operatorname{cn}(-\phi \xi)], \\
& F=2 \phi \operatorname{dn}(\phi \xi), \\
& W=U F, \quad \phi=\left[m\left(1-U^{2}\right)\right]^{-1 / 2},
\end{aligned}
$$

where $d n$ and $c n$ are the elliptic functions with the modulus $m$; $U$ and $m$ are constants $(|U|<1,0 \leq m \leq 1)$. We normalize in such a way that the period of $\theta$ is $2 \pi$, and find that

$$
k=\frac{\pi \phi}{2 K},
$$

where $K(m)$ is the complete elliptic integral of the first kind.

In terms of dislocations, the magnitude $N=k / 2 \pi$ is a linear density of dislocations, $U$ is velocity of dislocations. If $m=1$ the formulas (3) describe a one-soliton solution modeling one dislocation. The solution with $m<1$ is interpreted as an infinite succession of interacting dislocations. Let us average over a period of oscillation, the magnitudes of $F$ and $W$ :

$$
\begin{aligned}
& \bar{F}=\frac{\pi \phi}{2 K}, \\
& \bar{W}=U \bar{F} ;
\end{aligned}
$$

$\bar{F}$ and $\bar{W}$ can be interpreted as a force acting between dislocations, and a velocity of plastic deformation, respectively.

\section{SOLUTIONS OF THE SG EQUATIONS}

The solution procedure to be described below and the solutions themselves were described earlier ${ }^{16}$ in another form and for another purpose. For convenience and better understanding we repeat here the derivation of solutions.

\section{Modulated equations}

We limit our consideration to the wave train of type (3) of slow variation with the parameters $k, \omega, U$, and $m$. In this case, the solution of SG equation is described by the functions: $k(x, t), \omega(x, t), U(x, t)$, and $m(x, t)$. The equations describing the modulation of wave trains could be obtained by Whitham's variational method. ${ }^{15}$

Write the Lagrangian for the SG equation: $L=\left(\varphi_{t}^{2} / 2\right)-\left(\varphi_{x}^{2} / 2\right)-V(\varphi)$. For $\varphi=\varphi(\theta)$ we find $L=\left(\omega^{2}-k^{2}\right) \varphi_{\theta}^{2} / 2-V(\varphi)$. Average $L$ on the oscillation period using (2):

$$
\begin{aligned}
\bar{L} & =\frac{1}{2 \pi} \int L d \theta \\
& =\frac{1}{2 \pi}\left[2\left(\omega^{2}-k^{2}\right)\right]^{1 / 2} \int(A-V)^{1 / 2} d \varphi-A .
\end{aligned}
$$

Now suppose that variables $\omega, k$, and $A$ are slowly changing functions of $x$ and $t$, compared to the fast variable $\theta(x, t)$. These slow variables are described by the following equations: ${ }^{15}$

$$
\begin{aligned}
& \bar{L}_{A}=0, \\
& \bar{L}_{\omega, t}-\bar{L}_{k, x}=0, \\
& k_{t}+\omega_{x}=0 .
\end{aligned}
$$

The SG equation is second order, so the number of independent variables is only two. Using (4), (6), and (7) we obtain modulated equations in the shape

$$
\begin{aligned}
& U_{t} \frac{\mu}{U^{2}-1}+m_{t} \frac{U}{2 m}+U_{x} \frac{U \mu}{U^{2}-1}+m_{x} \frac{1}{2 m}=0, \\
& U_{t} \frac{U}{U^{2}-1}+m_{t} \frac{\mu}{2 m m_{1}}+U_{x} \frac{1}{U^{2}-1}+m_{x} \frac{U \mu}{2 m m_{1}}=0,
\end{aligned}
$$

Here $m_{1}=1-m, \mu=E / K, E(m)$ is the complete elliptic integral of the second kind. This system reduces to the diagonal form: ${ }^{17}$

$$
\begin{aligned}
& r_{i, t}+V_{i}\left(r_{1}, r_{2}\right) r_{i, x}=0, \quad i=1,2, \\
& V_{1}\left(r_{1}, r_{2}\right)=\frac{U \mu+\operatorname{sgn}\left(r_{2}-r_{1}\right) \sqrt{m_{1}}}{\mu+U \operatorname{sgn}\left(r_{2}-r_{1}\right) \sqrt{m_{1}}}, \\
& V_{2}\left(r_{1}, r_{2}\right)=V_{1}\left(r_{2}, r_{1}\right) .
\end{aligned}
$$


The Rimannian invariants $r_{i}$ are related to $m$ and $U$ by

$$
U=\frac{1-16 \sqrt{r_{1} r_{2}}}{1+16 \sqrt{r_{1} r_{2}}}, \quad m=\frac{4 \sqrt{r_{1} r_{2}}}{\left(\sqrt{r_{1}}+\sqrt{r_{2}}\right)^{2}} .
$$

\section{Simple waves}

Here, we will find solutions in the shape of a simple wave, that is, when one invariant $r_{i}$ is constant. Let $r_{2}=\varepsilon / 16$ be constant and $r_{2}>r_{1}$ (case with $r_{1}>r_{2}$ describes a wave train in the opposite direction), then $r_{1}$ is determined by the Eq. (9) with $i=1$. In this case the solution depends on one variable only. It is convenient to express all variables in terms of $m$. Using (4), (5), ( $\left.9^{\prime}\right)$, and (10), we can find

$$
U=\frac{\rho-\varepsilon}{\rho+\varepsilon}
$$

where

$$
\begin{aligned}
& \rho=\frac{\left(1-\sqrt{m_{1}}\right)^{2}}{m}, \\
& V_{1} \equiv V=\frac{G-\varepsilon}{G+\varepsilon},
\end{aligned}
$$

where

$$
\begin{aligned}
& G=\rho \frac{\mu-\sqrt{m_{1}}}{\mu+\sqrt{m_{1}}}, \\
& k=\bar{F}=\frac{\pi(\rho+\varepsilon)}{2 K \sqrt{m \rho \varepsilon}}, \quad \omega=\bar{W}=\bar{F} U .
\end{aligned}
$$

Setting $r_{1}=r_{1}(m)$ in (9) we obtain an equation for $m$ :

$$
m_{t}+V(m) m_{x}=0 \text {. }
$$

The solution of (12) has the form $m=R[x-V(m) t]$, where $R$ is an arbitrary function equal to the initial value of $m$ at $t=0: m(x, 0)=R(x)$. It is obvious the $m$ is constant on the lines $x-V(m) t=D(m)$, where $D$ is the inverse function to $R$.

\section{Self-simulating simple wave}

If $D(m)=0$, the solution has the form

$$
\frac{x}{t}=V(m) \text {. }
$$

For the interpretation of this solution, it is convenient to use the $(x, t)$ plane (Fig. 1). The solution presents an area expanding in time limited by the lines $x / t=V(m=0)=V^{-}(m=0)=-1$ and $x / t=V(m=1)$ $=V^{+}(m=1)=(1-\varepsilon) /(1+\varepsilon)$. Here and below the indexes "+" and "- " designate the belonging of datum to the leading and rear edges, respectively. Along the lines $x / t=V(m)$ all magnitudes are constant. On the right side of line $x / t=V^{+}$the disturbances are absent $(\bar{W}=0, \bar{F}=0, N=0)$. The density of dislocations decreases from $N\left(x=x^{-}, t^{c}\right)=\infty$ to $N\left(x=x^{+}, t^{c}\right)=0$ along the line $t=t^{c}$ and increases from $N\left(x^{c}, t=t^{1}\right)=0$ to $N\left(x^{c}, t \rightarrow \infty\right) \rightarrow N\left(m^{0}\right)\left[m^{0}\right.$ is the value of $m$ which satisfies $V(m)=0$ ] along the line $x=x^{c}>0$ (see Fig. 1).

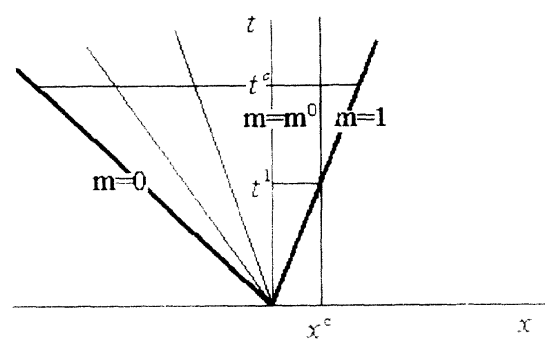

FIG. 1. Isolines of the function $m(x, t)$.

Below we will often use solutions with small values of $m_{1}\left(m_{1}^{+} \leq m_{1} \leq m_{1}^{-}\right.$, where $\left.m_{1}^{+}, m_{1}^{-} \ll 1\right)$. In this case, the parameter $2 \gamma=1-\varepsilon$ will also be small. This limitation allow us to simplify formulas (11):

$$
\begin{aligned}
& U=\gamma-m_{1}^{1 / 2}, \\
& V=\gamma-m_{1}^{1 / 2} \ln \left(m_{1}^{-1 / 2}\right), \\
& k=\bar{F}=\pi / \ln \left(m_{1}^{-1 / 2}\right), \\
& \omega=\bar{W}=\pi\left(\gamma-m_{1}^{1 / 2}\right) / \ln \left(m_{1}^{-1 / 2}\right) .
\end{aligned}
$$

Here we used the approximation $\ln \left(m_{1}^{-1 / 2}\right) \gg 1$. This term is equivalent to the term $2 N \ll 1$. It means that the dislocation density should be much less than atom density. This condition is frequently satisfied in real crystals.

\section{General solution}

The self-simulating solution satisfies the relation $\partial(m, U) / \partial(x, t)=0$. In the general case where $\partial(m, U) / \partial(x, t) \neq 0$, the system of Eq. (8) can be linearized using the hodograph method. ${ }^{18}$ Treating $x(m, U)$ and $t(m, U)$ as the unknowns, we transform (8) into the system:

$2 \mu m U t_{m}-2 \mu m x_{m}+\left(1-U^{2}\right) t_{U}-U\left(1-U^{2}\right) x_{U}=0$,

$2 m m_{1} t_{m}-2 m m_{1} U x_{m}$

$$
+\mu U\left(1-U^{2}\right) t_{U}-\mu\left(1-U^{2}\right) x_{U}=0 .
$$

Making the change of variable in (15),

$$
\begin{aligned}
& x=\frac{2 f U^{2}}{U^{2}-1}+\frac{g}{U-1}, \\
& t=\frac{2 f U^{2}}{U^{2}-1}+\frac{g U}{U-1},
\end{aligned}
$$

( $f$ and $g$ are new variables) we find

$g_{m} m \mu+f_{U} U(U-1)-\frac{f}{(1+U)}-\frac{g}{2}=0$,

$\frac{4 f_{m} m m_{1}}{\mu}+\frac{g_{U}(U+1)\left(U^{2}-1\right)}{U}-f-\frac{g(U+1)}{U}=0$.

In this system the variable can be separated. Suppose

$$
f=\boldsymbol{\Phi}(U) \boldsymbol{F}(m), \quad g=\Psi(m) \boldsymbol{G}(m) ;
$$

we obtain from (17) 


$$
\begin{aligned}
& U(1-U) \Phi^{\prime}=-\Phi /(1+U)+\alpha \Psi, \\
& (1+U)^{2}(1-U) \Psi^{\prime} / 4=\beta U \Phi-U \Psi, \\
& m m_{1} F^{\prime} / \mu=F / 2+\beta G, \\
& m \mu G^{\prime}=\alpha F+G / 2,
\end{aligned}
$$

where $\alpha$ and $\beta$ are arbitrary constants. Excluding $\Phi$ and $G$ from (19) and (20) we find the equations for $\Psi$ and $F$ :

$$
\begin{aligned}
(1+U)^{2}(1-U)^{2} \Psi^{\prime \prime} / 4+(1+U) & (1-U)^{2} \Psi^{\prime} / 2 \\
& +(1 / 4-\lambda) \Psi=0, \\
m^{2} m_{1} F^{\prime \prime}-m^{2} F^{\prime}+(1 / 4-\lambda) F= & 0,
\end{aligned}
$$

where $\lambda=\alpha \beta$.

The solution of Eq. (21) is

$$
\Psi=\eta^{q}, \quad \eta=(1-U) /(1+U),
$$

where $q$ satisfies the relation $(q-1 / 2)^{2}=\lambda$.

We limit our consideration of case $\lambda \geq 0$. From the second equation of system (19) we find

$$
\Phi=\frac{(1 / 2-q)(1+U) \eta^{q}}{2 U \beta}
$$

After transforming by $m=2 /(1+z)$ Eq. (22) reduces to a Legendre equation:

$$
\left(1-z^{2}\right) F^{\prime \prime}-2 z F^{\prime}+n(n+1) F=0,
$$

where $n(n+1)=\lambda-\frac{1}{4}$ or $n=\left|q-\frac{1}{2}\right|-\frac{1}{2}$. The solution of Eq. (25) and system (20) are

$F=L_{n}(z), \quad G=-\left[2 m_{1} L_{n}^{\prime}(z) / m \mu+L_{n}(z) / 2\right] / \beta$,

where $L_{n}(z)$ is some solution of the Legendre equation. Using Eqs. (16), (18), (23), (24), and (26) we finally find a one-parameter set of solutions:

$$
\begin{aligned}
& \chi=\left(x_{q}+t_{q}\right) / 2=a_{q}(m) \eta^{q-1}, \\
& \tau=\left(t_{q}-x_{q}\right) / 2=a_{1-q}(m) \eta^{q},
\end{aligned}
$$

where

$$
a_{q}=\frac{2 q L_{n}(z)}{(2 q-1)}+\frac{4 m_{1} L_{n}^{\prime}(z)}{(2 q-1) m \mu} .
$$

Consider the behavior of solution (27) for integer $q$ and for two invariants of $L_{n}: L_{n}=P_{n}$ and $L_{n}=Q_{n}$, where $P_{n}$ and $Q_{n}$ are the Legendre functions of the first and second kinds, respectively. For this purpose find isolines of the function $m(\chi, \tau)$ on plane $\chi, \tau$. Excluding $\eta$ from (27), we obtain

$$
\tau=c_{q}(m) \chi^{p},
$$

where $p=q /(q-1), c_{q}=a_{1-q} / a_{q}$. We can see from the relation (18) that isolanes $m(\chi, \tau)=$ const are parabolas of degree $p$. If $n$ increases $(n \rightarrow \infty)$, the degree of the parabolas, $p \rightarrow 1$, which corresponds to the self-simulated solution. So if we are restricted to the integer $q$, the most interesting solutions in sense of distinction from selfsimulated solutions are the solutions with $n=1$ (in this case $q=-1$ and $q=2$ ).
A periodic solution is found within the area restricted by lines with $m=0$ and $m=1$. In the case $q=-1$ $(p=1 / 2)$ and $L_{1}=P_{1}$, the leading edge accelerates in time. In any fixed point $x^{c}>0$ the dislocation density changes monotonically from $N\left(t<t^{c}\right)=0$ to $N(t \rightarrow \infty) \rightarrow \infty$ in contrast to the self-simulating wave solution where the density is limited as $t \rightarrow \infty$. In the case $q=2(p=2)$ and $L_{1}=Q_{1}$, the velocity of the leading edge is formally equal to unity; however, the dislocation density in any fixed point $x^{c}$ decreases in time $[N(t \rightarrow \infty) \rightarrow 0]$.

\section{INTERACTION OF GROUP OF DISLOCATIONS}

The solutions of the SG equation that we have obtained could be used for solving certain Cauchy problems. The imposed initial and boundary conditions should be constant if a simple wave solution is to be applied or monotone functions of $t$ or (and) $x$ could be posited, if a more general solution of the above class is used. More complicated initial and boundary conditions (periodic or nonmontone) are not suitably described by our formulas. This restriction is associated with our having considered only the form $\varphi=\varphi(\theta)$. We shall consider some examples of generation, propagation, and interaction of dislocations; our discussion will involve only the use of simple wave solutions.

\section{Generation of dislocations by source}

It is a well-known fact that the plasticity of crystals is realized to be not so much due to the movement of already available dislocations as due to newly formed dislocations. ${ }^{1}$ The sources of dislocations are some stress concentrators (such as tips of cracks, steps on the boundary, accumulation of contamination, etc.) and as well as dislocations themselves (for example, the widely known Frank-Read mechanism and its modifications ${ }^{1}$ ). So it is interesting to describe dynamic parameters of groups of dislocations in the presence of a given source.

First of all, we consider the case of dislocation generation in a crystal which initially does not contain dislocations $N(x>0, t=0)=0$. The action of an external stress as the source of dislocations is represented by the boundary condition $N(x=0, t \geq 0)=N^{0}$. It is necessary to describe the dynamics of this process. Putting in formulas (11) and (14) $k(0, t)=2 \pi N^{0}$ and $V(0, t)=0$ we find

$$
\varepsilon=G\left(m^{0}\right) \equiv G^{0},
$$

where $m^{0}$ is defined from the relation

$$
N^{0}=\frac{\mu\left(m^{0}\right)}{4 K\left(m^{0}\right)\left[m^{0}\left(\mu^{2}\left(m^{0}\right)-m_{1}^{0}\right)\right]^{1 / 2}},
$$

or

$$
m_{1}^{0}=e^{-1 / N^{0}}, \quad \gamma=e^{-1 / 2 N^{0}} / 2 N^{0}
$$

if $\ln \left(m_{1}^{-1 / 2}\right) \gg 1$. Formulas (11) and (13) with $\varepsilon$ and $m^{0}$ from (29), in the general case and formulas (13) and (14), with $\gamma$ and $m^{0}$ from (30), in the case when $\ln \left(m_{1}^{-1 / 2}\right) \gg 1$, describe the dynamics of dislocation 
propagation and interaction in the presence of a source.

Let us write the velocity of the leading edge which coincides with velocity of lead dislocation in this particular case:

$$
U^{+}=V^{+}=\frac{\left(1-G^{0}\right)}{\left(1+G^{0}\right)}
$$

or $U^{+}=V^{+}=e^{-1 / 2 N^{0}} / 2 N^{0}$, if $\ln \left(m_{1}^{-1 / 2}\right) \gg 1$.

Figure 2 schematically illustrates this process. The line $x / t=V^{+}$is the natural boundary between the area containing dislocations and the undisturbed area. Spatial distribution of density $N$, velocity of dislocations $U$, force of dislocation interaction $\bar{F}$, velocity of constant phase $V$, and velocity of plasticity $\bar{W}$ are presented for a fixed moment of time $t=t^{c}$ (see Fig. 2).

It is easy to rewrite the formulas (29)-(31) for the case when the given parameter is the velocity of plasticity deformation $W(0, t \geq 0)=W^{0}$. In this case $m^{0}$ is defined from relation

$$
W^{0}=\frac{\pi\left(m_{1}^{0}\right)^{1 / 2}}{K\left(m^{0}\right)\left[m^{0}\left(\mu^{2}\left(m^{0}\right)-m_{1}^{0}\right)\right]^{1 / 2}}
$$

or for $\ln \left(m_{1}^{-1 / 2}\right) \gg 1$,

$m_{1}^{0}=\left(W^{0} / \pi\right)^{2}, \quad U^{+}=V^{+}=\gamma=\left(W^{0} / \pi\right) \ln \left(W^{0} / \pi\right)^{-1}$.

Real crystals usually contain dislocations. Under the action of external stress these available dislocations move and simultaneously the sources of a new dislocation join in. To describe these processes we will consider the generation of dislocations in a crystal with a primary density $N(x>0, t=0)=N^{+}<N^{0}$ and velocity $U(x>0, t=0)$ $=U^{+}$. We can use the self-simulating simple wave solution for this task if only one of quantities $U^{+}$or $N^{+}$is independent. Let it be $N^{+}$. Then formulas (11), (13), (29),

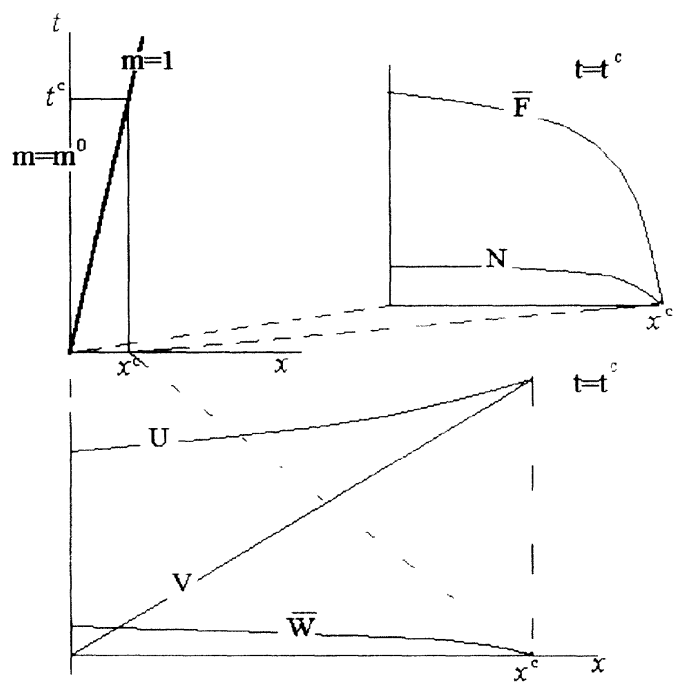

FIG. 2. Functions $\bar{F}\left(x, t^{c}\right), N\left(x, t^{c}\right), U\left(x, t^{c}\right), V\left(x, t^{c}\right)$, and $\bar{W}\left(x, t^{c}\right)$ for the case of a given dislocation source in the point $x=0$. and (32) in the general case or (13), (14), (30), and (33) in the case $\ln \left(m_{1}^{-1 / 2}\right) \gg 1$ describe process. However, in contrast with the previous case, the leading edge is defined by the value $m=m^{+}$(instead of $m=1$ ), where $m^{+}$is related to $N^{+}$by

$$
N^{+}=\frac{\mu^{+}}{\left.4 K\left(m^{+}\right)\left[m^{+}\left(\mu^{+}\right)^{2}-m_{1}^{+}\right)\right]^{1 / 2}}, \mu^{+} \equiv \mu\left(m^{+}\right) \text {, }
$$

or for $\ln \left(m_{1}^{-1 / 2}\right)>1$,

$$
m_{1}^{+}=e^{-1 / N^{+}} \text {. }
$$

The velocity of the leading edge is

$$
\begin{aligned}
& V^{+}=\left(G^{+}-G^{0}\right) /\left(G^{+}+G^{0}\right), G^{+} \equiv G\left(m^{+}\right), \\
& V^{+}=\left(1 / 2 N^{0}\right) e^{-1 / 2 N^{0}}-\left(1 / 2 N^{+}\right) e^{-1 / 2 N^{+}}
\end{aligned}
$$

where we used $\ln \left(m_{1}^{-1 / 2}\right) \gg 1$ in the last formula.

Magnitude $x^{+}=V^{+} t$ defines the size of the plasticity zone. Using these relations, it is easy to connect the size of the plasticity zone with the power of the source or a given velocity of deformation on the boundary.

\section{Diffusion of dislocations}

Real crystals practically always contain internal stress and as a result of it the irregular distribution density occurs. Since this state is unstable the internal stresses aspire to relax. This process could be realized by diffusion of dislocations. Consider the following situation. Let the plane $x=0$ divide areas with different density and velocity of dislocations at time $t=0$ :

$$
\begin{aligned}
& N(x<0, t=0)=N^{-}, \quad U(x<0, t=0)=U^{-}, \\
& N(x>0, t=0)=N^{+}<N^{-}, \quad U(x>0, t=0)=U^{+} .
\end{aligned}
$$

What will happen when $t>0$ ? Note that only three of the four quantities $N^{-}, N^{+}, U^{-}$and $U^{+}$can be considered independent to make use of the simple wave solution. Let us suppose that $U^{+}$depends on the others. Then the behavior of the system is described by formulas (11) and (13) with

$$
\varepsilon=\rho\left(m^{-}\right) \frac{\left(1-U^{-}\right)}{\left(1+U^{-}\right)},
$$

where $\mathrm{m}^{-}$is defined by the relation

$$
N^{-}=\left\{4 K\left(m^{-}\right)\left[m^{-}\left(1-\left(U^{-}\right)^{2}\right)\right]^{1 / 2}\right\}^{-1},
$$

or formulas (13) and (14) with

$$
\gamma=U^{-}+e^{-1 / 2 N^{-}}, m_{1}^{-}=e^{-1 / N^{--}},
$$

if $\ln \left(m_{1}^{-1 / 2}\right) \gg>1$. Formulas for the velocity of the leading and rear edges are

$$
\begin{aligned}
& V^{-}=\left(G^{-}-\varepsilon\right) /\left(G^{-}+\varepsilon\right), \quad G^{-}=G\left(m^{-}\right), \\
& V^{+}=\left(G^{+}-\varepsilon\right) /\left(G^{+}+\varepsilon\right),
\end{aligned}
$$

where $m^{+}$is defined by the relation 


$$
N^{+}=\frac{\rho^{+}+\varepsilon}{4 K\left(m^{+}\right)\left(m^{+} \rho^{+} \varepsilon\right)^{1 / 2}}, \quad \rho^{+}=\rho\left(m^{+}\right)
$$

with $\varepsilon$ from (37). If $\ln \left(m_{1}^{-1 / 2}\right) \gg 1$ formulas (39) reduce to

$$
\begin{aligned}
& V^{-}=U^{-}-\left(2 N^{-}\right)^{-1} e^{-1 / 2 N^{-}}, \\
& V^{+}=U^{-}+e^{-1 / 2 N^{-}}-\left(2 N^{+}\right)^{-1} e^{-1 / 2 N^{+}} .
\end{aligned}
$$

Formula (39) [or (41)] is convenient for the evaluation of the typical time of internal stress relaxation.

Spatial distribution of considered parameters in some moment $t=t^{c}$ is presented in Fig. 3. We can see from the figure and formulas (41) that $\left|V^{-}\right| \gg\left|V^{+}\right|$and $\left|x^{-}\right| \gg\left|x^{+}\right|$if $N^{-} \gg N^{+}$and $U^{-}=0$, e.g., a disturbance propagates faster in the direction with larger dislocation density. In particular, it means that as a result of dislocation diffusion through boundaries of some region of high dislocations density, the pileup slowly spreads. During the same time, the dislocation density decreases slowly but almost uniformly along the pileup. This behavior of the FK chain is not evident before this analysis.

\section{Model of crack}

The appearance and development of cracks is an important cause of crystal materials destruction. In relation with this, dislocation-crack interactions have been widely studied. ${ }^{19}$

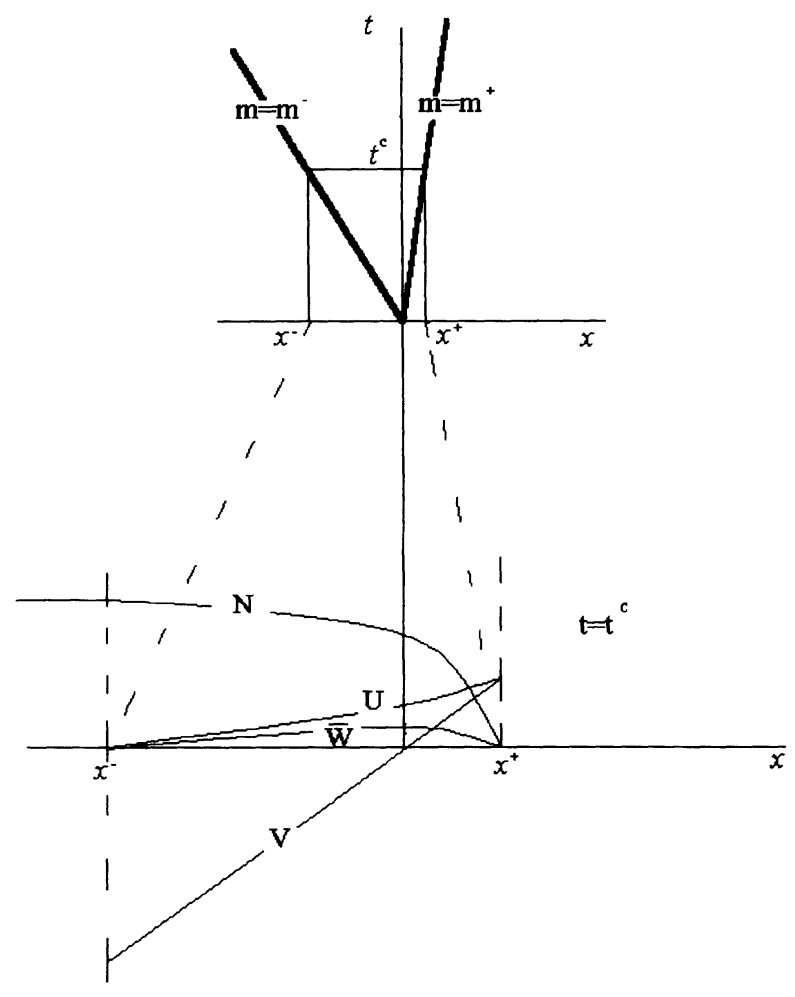

FIG. 3. Functions $N\left(x, t^{c}\right), U\left(x, t^{c}\right), V\left(x, t^{c}\right)$, and $\bar{W}\left(x, t^{c}\right)$ for the case of dislocation diffusion.
One of the possible cause of crack origin is the accumulation of dislocations near natural obstacles (boundaries of grains, accumulations of contamination, etc.). ${ }^{1}$ Increasing the dislocation density leads to an increase in the energy of their interaction. If some threshold value is exceeded, the equilibrium state becomes unstable and the energy of dislocation interactions transforms to the energy of the movement of crystal parts along a glide plane (this corresponds to propagation of crack mode-II). Dynamic parameters of this process could be described by formulas (11), (13), (37), and (39) [or formulas (13), (14), (38), and (40) for small values of $\left.m_{1}\right]$ with $U^{-}=0$. In this case magnitude $V^{-}$is the velocity of the crack tip.

Now consider a dislocation nucleation from the moving crack tip. It is known that crack tip is a stress concentrator. If as a result of the increase in external stress the shear stress at the crack tip exceeds the threshold magnitude, the crack will begin to increase. Its tip will be the source of dislocations. Supposing that in the moment $t=0$, the crack is at the left side of the plane $x=0$, we give following magnitudes: velocity of the tip $V^{\mathrm{cr}}=V\left(x^{\mathrm{cr}}, t>0\right)=x^{\mathrm{cr}} / t>0$, where $x^{\mathrm{cr}}$ is coordinate of tip; the force which generates dislocation on the tip $\bar{F}\left(x^{\mathrm{cr}}, t>0\right)=F^{\mathrm{cr}}$, and initial the dislocation density on the right side of the crack tip $N(x>0, t=0)=N^{+}$. Then we find from (11)

$$
\varepsilon=G\left(m^{\mathrm{cr}}\right)\left(1-V^{\mathrm{cr}}\right) /\left(1+V^{\mathrm{cr}}\right),
$$

where $m^{\mathrm{cr}}$ connects with $V^{\mathrm{cr}}$ and $F^{\mathrm{cr}}$ by the relation

$$
\begin{gathered}
F^{\mathrm{cr}}=\frac{\pi\left[\mu^{\mathrm{cr}}+\left(m_{1}^{\mathrm{cr}}\right)^{1 / 2} V^{\mathrm{cr}}\right]}{K\left(m^{\mathrm{cr}}\right)\left\{m^{\mathrm{cr}}\left[\left(\mu^{\mathrm{cr}}\right)^{2}-m_{1}^{\mathrm{cr}}\right]\left[1-\left(V^{\mathrm{cr}}\right)^{2}\right]\right\}^{1 / 2}}, \\
\mu^{\mathrm{cr}} \equiv \mu\left(m^{\mathrm{cr}}\right) .
\end{gathered}
$$

The velocity of the leading edge is

$$
V^{+}=\left(G^{+}-\varepsilon\right) /\left(G^{+}+\varepsilon\right) \text {, }
$$

where $\mathrm{m}^{+}$is defined from (40) with $\varepsilon$ from (42).

The velocity of dislocations on the rear edge (at the top) is

$$
U^{-}=\frac{\mu^{\mathrm{cr}} V^{\mathrm{cr}}+\left(m_{1}^{\mathrm{cr}}\right)^{1 / 2}}{\mu^{\mathrm{cr}}+V^{\mathrm{cr}}\left(m_{1}^{\mathrm{cr}}\right)^{1 / 2}}>V^{\mathrm{cr}} .
$$

Note that $U^{-}=\left(m_{1}^{\text {cr }}\right)^{1 / 2} / \mu^{\text {cr }}>0$ if $V^{\text {cr }}=0$, e.g. the top emits dislocations even though the crack does not grow up.

If $F^{\mathrm{cr}} \ll \pi$ [or $\ln \left(m_{1}^{-1 / 2}\right) \gg 1$ ] formulas (43)-(45) could be essentially reduced:

$$
\begin{aligned}
& m_{1}^{\mathrm{cr}}=e^{-2 \zeta}, \quad \xi=\frac{\pi}{F^{\mathrm{cr}}\left(1-\left(V^{\mathrm{cr}}\right)^{2}\right)^{1 / 2}}, \\
& \delta U^{-} \equiv U^{-}-V^{\mathrm{cr}}=V^{\mathrm{cr}}\left(1-\left(V^{\mathrm{cr}}\right)^{2}\right) \zeta e^{-\zeta}, \\
& \delta V^{+} \equiv V^{+}-V^{\mathrm{cr}}=2 V^{\mathrm{cr}}\left(1-V^{\mathrm{cr}}\right) \zeta e^{-\zeta} .
\end{aligned}
$$

We can see from the last formulas that the relative velocities of emitted dislocations $\partial U^{-}$and leading edge $\delta \mathrm{V}^{+}$decrease with increasing crack tip velocity. The important parameters as a size of the plasticity zone in front 
of the crack tip and density and velocity of formed dislocations could be evaluated by the obtained formulas.

\section{CONCLUSION}

The FK model is a simple and convenient apparatus for studying dislocation. We developed this model for the description of dynamic behavior of a large number of interacting dislocations. For this purpose we obtained solutions of Whitham's modulated equations based on SG equations, which is a continuum limit of the FK model. As a result of this, it was possible to find the relations connecting plasticity parameters and dislocation parameters (density, velocity, and force acting between dislocations). In particular, this model naturally connects three important velocities: the sound velocity of the crystal lattice, the velocity of dislocation movement, and the velocity of plasticity along the glide plane. Formulas obtained allowed us to describe spatial and temporal distribution of dislocations for different initial and boundary conditions. As examples of this application we modeled some nonstationary processes, namely,

(i) the generation of dislocations due to a given source or given deformation; (ii) the diffusion of dislocations due to self-interaction, if the initial spatial distribution is inhomogeneous;

(iii) the generation of dislocations by moving the crack tip.

These results could be used for the estimation of the power of sources, typical size of the plasticity zone, typical time of local internal stress relaxation, the relationship between velocity of crack tip and number, density and velocity of that generated in front of the tip dislocations.

Our choice of examples was based on the simple wave solution of the SG equation, which was able to describe processes with constant initial and boundary conditions. More general solutions which we derived above could be used to model more complicated regimes with the monotone changing initial and boundary parameters.

\section{ACKNOWLEDGMENTS}

The author gratefully acknowledges $\mathbf{A}$. Krylov and $\mathbf{N}$. Mazur for many fruitful discussions, T. Svobodny for a critical reading of the manuscript, and the cooperation of the Physics Department of Wright State University.
1J. P. Hirth and J. Lothe, Theory of Dislocations (Wiley, New York, 1982).

2J. Frenkel and T. Kontorova, J. Phys. (Moscow) 1, 137 (1939).

${ }^{3}$ F. C. Frank and J. H. van der Merwe, Proc. R. Soc. London, Ser. A 198, 205 (1949); 198, 216 (1949); 200, 125 (1950); 201, 261 (1950).

${ }^{4}$ V. L. Indenbom, Soviet Phys. Crystallogr. 3, 193 (1958).

${ }^{5}$ R. Hobart, J. Appl. Phys. 36, 1944 (1965); 36, 1948 (1965).

${ }^{6}$ A. Seeger and Schiller, in Physical Acoustics, edited by W. P. Mason (Academic, New York, 1966), Vol. III.

${ }^{7}$ Y. Y. Earmme and J. H. Weiner, Phys. Rev. Lett. 33, 1550 (1974).

${ }^{8}$ N. Flytzanis, S. Crowley, and V. Celli, Phys. Rev. Lett. 39, 891 (1977).

${ }^{9}$ A. Milchev, Phys. Rev. B 42, 6727 (1990); A. Milchev, Th.

Fraggis, and St. Pnevmatikos, Phys. Rev. B 45, 10348 (1992).

${ }^{10}$ Y. Hashizume, J. Phys. Soc. Jpn. 62, 2241 (1993).

${ }^{11}$ M. Peyrard and Kruskal, Physica 14D, 88 (1984).

${ }^{12}$ O. M. Braun and Y. S. Kivshar, Phys. Lett. A 149, 119 (1990); Phys. Rev. B 43, 1060 (1991).

${ }^{13}$ T. Munakata, Phys. Rev. A 45, 1230 (1992).
${ }^{14}$ A. Barone, F. Esposito, C. J. Magee, and A. C. Scott, Nuovo Cimento 1, 227 (1971); A. C. Scott, F. Y. F. Chu, and D. W. McLaughlin, Proc. IEEE 61, 1443 (1973); in Solitons in Action, edited by $\mathbf{K}$. Lonngren and A. Scott (Academic, New York, 1978); L. Lamb, Jr., Elements of Soliton Theory (Wiley, New York, 1980); in Solitons, edited by S. E. Trullinger, V. E. Zakharov, and V. L. Pokrovski (North-Holland, Amsterdam, 1986).

${ }^{15}$ G. B. Whitham, Proc. R. Soc. London, Ser. A 293, 238 (1965); Linear and Nonlinear Waves (Wiley, New York, 1974).

${ }^{16}$ A. V. Gurevich, N. I. Gershenzon, A. L. Krylov, and N. G. Mazur, Sov. Phys. Dokl. 34(3), 246 (1989).

${ }^{17}$ M. G. Forest and D. W. McLaughlin. Stud. Appl. Math. 68, 11 (1983).

${ }^{18}$ L. D. Landau and E. M. Lifshitz, Fluid Mechanics (Pergamon, Oxford, 1959).

${ }^{19}$ R. Thomson, J. Mater. Sci. 13, 128 (1978); A. C. Eringen, J. Appl. Phys. 54(12), 6811 (1983); S. M. Ohr, Scr. Metall. 21, 1681 (1987); L. M. Brock and J.-S. Wu, J. Appl. Mech. 56, 572 (1989); J. R. Rice, J. Mech. Phys. Solids 40(2), 239 (1992). 